\title{
Positive discrimination contrast with delay of reward or low drive
}

\author{
RICHARD S. CALEF and RUTH ANN B. CALEF \\ West Virginia Wesleyan College, Buckhannon, West Virginia 26201 \\ FREDERICK R. MAXWELL \\ Southwest Missouri State University, Springfield, Missouri 65302
}

and

EARL R. McHEWITT

King College, Bristol, Tennessee 37620

\begin{abstract}
In the present study, six groups of 10 albino rats each were given differential conditioning training in parallel alleys differing only in brightness. The results showed a positive $S+$ contrast effect when 20 -sec delay of reinforcement or lowered drive (12-h deprivation) were used to lower responding, but a negative S+ contrast effect when subjects received both immediate reinforcement and 23-h deprivation. No present theory can adequately explain both effects.
\end{abstract}

Previous alley studies have shown that performance of rats to the negative discriminandum (S-) in differential conditioning is influenced by the reinforcement contingencies associated with the positive discriminandum (S+) (see Black, 1968; McHose, 1970, for reviews of the literature). Such studies have demonstrated that the $S$ - performance of differentially reinforced (discrimination) groups is depressed relative to that of a control group receiving the smaller reward in both the "S+" and "S-" (negative S- contrast). Until recently, however, positive $S+$ contrast has seldom been observed in the discrimination contrast literature. Previous studies have shown the St performance of discrimination groups to be depressed (Henderson, 1966; MacKinnon, 1967) relative to control groups receiving the larger reward in both discriminanda (negative $\mathrm{S}+$ contrast effect). These findings are particularly nonsupportive of perceptual theories (Dunham, 1968; Helson, 1964) which treat reward magnitude mainly in terms of its stimulus properties and predict symmetrical contrast effects (negative $\mathrm{S}$ - and positive $\mathrm{S}+$ contrast).

According to Mellgren, Wrather, and Dyck (1972), previous failures to find positive $S+$ contrast (Bower, 1961) may have occurred because the large reward control groups were displaying extremely rapid speeds that were approximately the physiological limits of the organism ("ceiling" effect). Mellgren et al. (1972) demonstrated positive S+ contrast using delay of reward to prevent running speeds from reaching a "ceiling" and concluded that previous failures to obtain the effect were an artifact of these designs. However, their results

This paper is sponsored by James McHose, who takes full editorial responsibility for its contents. The authors wish to express thanks to Chris Mundy for typing the manuscript and to Irving Calef, the first author's father, for constructing the apparatus. need not necessarily be attributed to removing the response "ceiling" but might be a result peculiar to delay of reinforcement. For example, to "wait" for a reward may accentuate the perception of its magnitude or reinforcement value, or, as Benefield, Oscos, and Ehrenfreund (1974) have suggested, the delay may produce frustration, and the positive contrast may result from the subjects being shifted from frustratingly small to larger incentives. In addition, Mellgren et al. (1972) employed a brightness-correlated startbox and alley section in order to control for time consumed during a "decision-like process" postulated for the experimental group. Also, Mellgren et al. (1972) employed an alternation schedule in the sequencing of positive and negative trials, a procedure not generally used in the discrimination literature and unknown in its effects on positive or negative contrast phenomena. Lastly, Mellgren et al. (1972) did not include a regular condition (immediate reward, 23-h deprivation). It is possible that the distinct procedures employed by the authors may have produced positive contrast in the regular condition. In any case, these manipulations preclude determination of the conditions sufficient for obtaining positive S+ contrast and present difficulties in interpreting the Mellgren et al. (1972) data.

The purpose of the present study was to determine the conditions sufficient for obtaining positive contrast. In the present study, an attempt was made to lower responding by reducing drive level or delaying reinforcement. A regular condition (immediate reward, 23-h deprivation) was used to enhance interpretation of the data. If positive contrast is not peculiar to delay of reinforcement, but, rather, is obtainable under different procedures having in common, seemingly, only lowered response speeds, then previous failure to demonstrate positive S+ contrast might reasonably be attributed to 


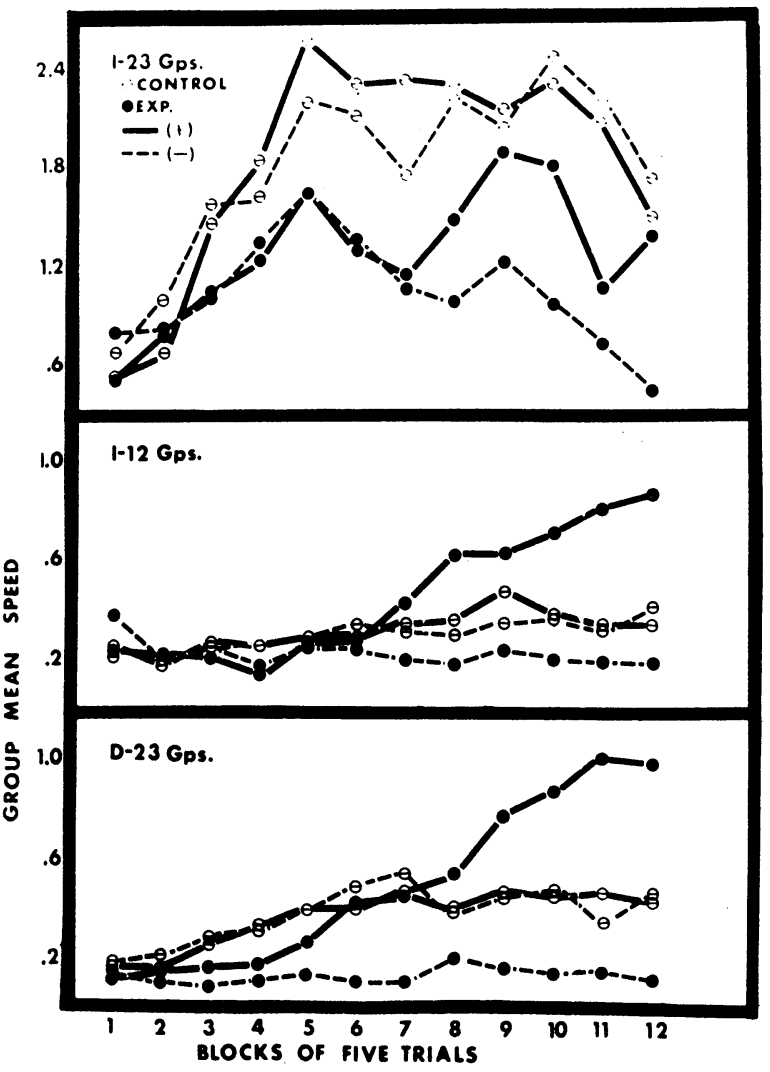

Figure 1. Speeds to the positive and negative discriminada for the experimental and control groups as a function of blocks of five trials per discriminandum.

the rapid response speeds produced in these experiments.

\section{METHOD}

\section{Subjects}

A total of 60 naive male albino rats, bred at West Virginia Wesley an College, were randomly divided in to six groups of 10 subjects each.

\section{Apparatus}

The runway apparatus was essentially the same as that described by Ludvigson \& Gay (1967) escept that only two of the multiple parallel alleys were used. The alleys comprised a $33.02-\mathrm{cm}$ gray startbox, two $66.04-\mathrm{cm}$ run sections, and two $30.48-\mathrm{cm}$ goalboxes, one painted flat black and the other flat white. The inner width and height of each section of the runways was $7.62 \mathrm{~cm}$. Photocell clock circuitry provided independent traversal times over the first $20.32-\mathrm{cm}$ segment (start time) and the next $30.48-\mathrm{cm}$ segment (run time) of each runway. A solenoid-operated opaque guillotine-ty pe retrace door separated alley-goal sections of the runways. The entire apparatus was covered with an opaque Plexiglas ceiling.

\section{Preliminary Training}

Twelve days prior to the 1st day of training (Day 13), all subjects were placed on an appropriate deprivation schedule, which was main tained throughout the study. On Day s 6-12, the rats were taken from their individual home cages and handled for about 5 min each. On Days 11-12, subjects were also placed in individual carrying cages and taken to the experimental room where they were allowed to explore the start and run sections of the apparatus while clocks, photocells, and doors were operated. On these. 2 days, approximately $1 \mathrm{~g}$ of $45-\mathrm{mg}$ Noyes pellets, identical to the subsequent reinforcement pellets, was incorporated into the subjects' daily food ration.

\section{Experimental Design and Procedure}

All experimental (E) groups received eight pellets in the S+ discriminandum and one pellet in the $S$ - discriminandum. All control (C) groups received eight pellets in both discriminanda, one of which was arbitrarily designated $S+$ and the other $S-$ for purposes of trial position control and ease of analysis. In all groups, half of the subjects were randomly assigned a black S+ and half a white S+. Groups ED23 and CD23 received a 20-sec deprivation schedule during the course of the experiment. Groups EI12 and CI12 received immediate reinforcement, but were maintained on a 12-h deprivation schedule $(12 \mathrm{~h}$ on food and $12 \mathrm{~h}$ off food). Finally, Groups EI23 and CI23 were treated in the standard fashion, e.g., received immediate reinforcement and maintained on a $23-\mathrm{h}$ deprivation schedule $(1 \mathrm{~h}$ of free food).

Four trials a day, 2 black and 2 white counterbalanced for daily trial position over successive 6 -day blocks of trials, were administered to five squads of 12 subjects each, comprised of two rats from each group. The running order of subjects within a squad was randomized from day to day. Each rat within a squad received his first daily trial before any subject received a second trial. The intertrial interval was approximately $7 \mathrm{~min}$. Throughout the experiment, the startdoor was opened following a 3-sec orientation (the clock started when the door was opened) and the retrace door was closed as soon as the rat entered the goalbox.

\section{RESULTS}

Since start and run speeds produced essentially similar results, only the start speeds will be presented here due to their higher levels of statistical reliability. Group mean start speeds as a function of blocks of five trials per discriminandum (a total of 120 trials) for all groups are presented in Figure 1. Each experimental group and its control is plotted separately. Speeds to the S+ and Sdiscriminanda are also plotted separately for each group.

As may be seen in Figure 1, each experimental group developed differential speeds to the S+ and $\mathrm{S}-$ discriminanda over the course of the experiment, while none of the control groups showed differences of any magnitude. Statistical analyses support these observations. Separate $2 \times 2 \times 2 \times 2$ analyses of variance were computed for each experimental group and its appropriate control with brightness of $\mathrm{S}+$ (white vs. black), early blocks $(1,2,3)$ vs. late blocks $(10,11,12)$ acquisition speeds, and $S+$ speeds vs. $S-$ speeds as factors. In all three cases, significant $(p<.05)$ Groups by Blocks by $\mathrm{S}+$ vs. S- interactions were obtained, $F(1,16)=7.59,74.71$, and 29.9 for the I-23, I-12, and D-23 groups, respectively. Subsequent paired comparisons using the Tukey (a) procedure on late acquisition totals established significant $(p<.05)$ discrimination performance in each experimental group, while $t$ tests indicated no differences $(p>.10)$ in control group speeds to the $\mathrm{S}+$ and $\mathrm{S}$ - discriminandum.

Turning to S+ speeds, it can be seen in Figure 1 that the $\mathrm{S}+$ speeds of the experimental I-23 group were below 
those of its control over the later stages of training, whereas the $\mathrm{S}+$ speeds of the experimental I-12 and D-23 groups were well above those of their controls. Paired comparisons of late acquisition speeds using the Tukey (a) procedure supported these graphic observations $(\mathrm{p}<.05)$.

\section{DISCUSSION}

The present data demonstrate that positive discrimination contrast is not less genuine than negative discrimination contrast. This observation is of particular interest since the two most recent major reviews of the literature (Black, 1968; McHose, 1970) have concluded that positive discrimination contrast does not occur and have proposed theoretical or descriptive models which predict no positive contrast effects.

It is noteworthy that, within the present study, both negative and positive $S+$ contrast were observed. A negative $S+$ contrast effect was found under conditions of immediate reward and high drive, conditions which yield extremely rapid response speeds. A positive S+ contrast effect was found under conditions of delayed reward or low drive which are known to yield speeds considerably below the limits of the organism. These findings, then, would imply that the positive S+ contrast observed here and those reported by Mellgren et al. (1972) were probably due to lowered response speeds rather than any "interaction" of delay of reward and magnitude shifts, frustration engendered by the delay, or "decision-like processes" interfering with responding in the experimental groups. Given the above analysis, another manipulation which would be expected to result in an observed positive $\mathrm{S}+$ contrast effect would be the introduction of some form of punishment into the goal region, thereby suppressing runway speeds. Indeed, Shanab and White (1972) have reported positive $S+$ contrast within a successive contrast (Crespi shift) paradigm using just such a procedure.

Frustration-based theories (Black, 1968) or pure descriptive models (McHose, 1970) designed to predict both negative Sand negative $S+$ discrimination contrast would seem to be inadequte in light of these and other similar findings (e.g., Mellgren et al., 1972). The present data are also inconsistent with the perceptual theories (Dunham, 1968; Helson, 1964) which predict symmetrical effects for contrasted conditions of reward, e.g., positive $S+$ contrast and negative $S-$ contrast but not the occurrence of negative $S+$ contrast under conditions of immediate reward high drive. Perceptual theory could explain the literature if the negative S+ contrast effect were seen merely as an artifact of the ceiling effect (Mellgren et al., 1972). However, no effect at all (Bower, 1961) could more justifiably be seen as an artifact of a ceiling effect. In the present study, for the experimental group to be significantly below the control on $S+$ trials should indicate that the negative $S+$ contrast effect is legitimate and not just an artifact of the ceiling effect. Further, it is almost axiomatic that an emotional explanation of positive S+ contrast cannot be ruled out. To wit, experience with contrasting magnitudes of reward may produce "elation" or "excitation" when in the presence of the stimulus associated with the larger magnitude of reward. Since a control group receiving only the large reward does not experience contrasted rewards, "elation" or "excitation" should not be experienced. Assuming that "positive emotional states" lead to heightened responsiveness and performance, the group receiving contrasted conditions of reward should demonstrate positive $S+$ contrast. It should be reiterated, however, that none of these models (emotional, perceptual, or descriptive) offer any rationale obvious to these authors for both the negative and positive $\mathrm{S}+$ contrast effects observed in this study, nor do we have, at present, a ready alternative.

It should also be mentioned that the present results are inconclusive as regards linking simultaneous contrast effects (discrimination contrast) and successive contrast effects (Crespi shifts). Delay of reward has been used successfully to demonstrative positive $S+$ contrast in the runway in both the Crespi shift (Shanab, Sander, \& Premack, 1969) and discrimination (Mellgren et al., 1972) paradigms. However, while lower drive level resulted in positive $S+$ contrast in the present study within a discrimination contrast paradigm, no positive contrast was reported using a similar drive manipulation in a Crespi shift paradigm (Ehrenfreund, 1971). Thus, there does not seem to be any discernable theoretical or empirical approach which can incorporate all of the data within one of these experimental paradigms, and certainly there is even less understanding of their relationships and underlying mechanisms. The best summary statement regarding simultaneous $\mathrm{S}+$ contrast would seem to be that conditions producing rapid responding produce negative $S+$ contrast, while conditions producing slower responding result in positive $S+$ contrast.

\section{REFERENCES}

Benefield, R., Oscos, A., \& Ehrenfreund, D. Role of Frustration in successive positive contrast. Journal of Comparative and Physiological Psychology, 1974, 86, 648-651.

Black, $R$. W. Shifts in magnitude of reward and contrast effects in instrumental and selective learning: $A$ reinterpretation. Psychological Review, 1968, 75, 114-126.

Bower, G. H. A contrast effect in differential conditioning Journal of Experimental Psy chology, 1961, 62, 196-199.

Dunham, P. J. Contrasted conditions of reinforcement: A selective critique. Psy chological Bulletin, 1968, 69, 295-315. Ehrenfreund, D. Effect of drive on successive magnitude shift in rats. Joumal of Comparative and Physiological Psychology, $1971,76,418-423$.

Helson, H. Adaptation-level theory: An experimental and systematic approach to behavior. New York: Harper and Row, 1964.

Henderson, K. Within-subjects partial reinforcement effects in acquisition and in later discrimination training. Journal of Experimental Psychology, 1966, 72, 704-713.

Ludvigson, H. W., \& Gay, R. A. An investigation of conditions determining contrast effects in differential reward conditioning. Journal of Experimental Psychology, 1967, 75, 37-42.

MacKinnon, J. R.

Interactive effect of the two rewards in a differential magnitude of reward discrimination. Journal of Experimental Psy chology, 1967, 75, 329-338.

McHose, J. H. Relative reinforcement effects: $S_{1} / S_{2}$ and $S_{1} / S_{1}$ paradigms in instrumental conditioning. Psy chological Review, $1970,77,135-146$.

Mellgren, R. L., Wrather, D. M., \& Dyck, D. G. Differential conditioning and contrast effects in rats. Journal of Comparative and Physiological Psychology, 1972, 80, 478-483.

Shanab, M. E., Sander, R.. \& Premack, D. Positive contrast in the runway obtained with delay of reward. Science, 1969, 164, 724-725.

Shanab, M. E., \& White, R. Positive contrast obtained with punishment. Journal of General Psychology, 1972, 86, 247-251.

(Received for publication March 29, 1975.) 